\title{
Change of Strength of Recycled Concrete with Age
}

\author{
Jiankui Zhang, Xian Ding \\ Sichuan Engineering Technical College, Deyang, Sichuan, China
}

\begin{abstract}
:
In this paper, a mathematical model of the relationship between the amount of various components of recycled concrete and its compressive strength is established by using a large number of experimental data on the strength performance of recycled concrete and BP artificial neural network technology. In this paper, the influence trend of water binder ratio and aggregate substitution rate on the strength of recycled concrete is studied by this model. Taking recycled concrete with different recycled aggregate as the research object, a nonlinear structural model reflecting the mapping relationship between mix proportion and strength of recycled concrete is established in this paper. In this paper, the predicted values of the model are compared with the experimental data. The research shows that the strength model of recycled concrete based on BP neural network established in this paper can predict the compressive strength of recycled concrete at the corresponding age according to the mix proportion of recycled concrete, and the prediction accuracy is high. The application of BP neural network effectively reduces the times of a large number of repeated mix proportion test when preparing recycled concrete in practical engineering, reduces the waste of human and material resources, and has good economic benefits.
\end{abstract}

Keywords: Recycled Concrete, Strength Performance, BP Neural Network, Compressive Strength.

\section{INTRODUCTION}

At present, China is at the peak of the wave of infrastructure construction. In the next few decades, concrete will still be the most widely used building material. With the wide application of concrete, the demand for sand aggregate, the main component of concrete, is increasing [1-2]. As we all know, the relationship between the mix proportion and strength of ordinary concrete can be realized by boromi formula [3]. However, the aggregate source of recycled concrete is complex, the relationship between unit water consumption of recycled concrete and its working performance is uncertain, and the relationship between compressive strength and water binder ratio of recycled concrete is also uncertain [4-5]. Because BP neural network has the 
Article History: Received: 28 October 2021 Revised: 05 December 2021 Accepted: 10 January 2022 Publication: 28 February 2022

characteristics of large-scale parallel, distributed storage and processing, self-organization, selfadaptive and self-learning ability, it is especially suitable for dealing with uncertain and fuzzy information processing problems that need to consider many factors and conditions at the same time. Therefore, this paper will apply BP artificial neural network technology to study the mechanical properties of recycled coarse aggregate concrete and recycled fine aggregate concrete, and establish the nonlinear relationship between the content of recycled concrete raw materials and its compressive strength [6]. That is, the compressive strength of recycled concrete at the corresponding age can be accurately predicted according to the content of raw materials in each part of recycled concrete. At the same time, the strength prediction model of recycled concrete based on BP neural network can be used to analyze and study the influence trend of water binder ratio and aggregate substitution rate on the compressive strength of recycled concrete.

\section{INFLUENCING FACTORS OF GREEN CONCRETE STRENGTH}

\section{Effect of water binder ratio on mechanical properties of recycled concrete}

For ordinary concrete, water binder ratio is the most important factor affecting its compressive strength. Generally speaking, the compressive strength of concrete is inversely proportional to the water binder ratio [7-8]. The lower the water binder ratio, the higher the compressive strength. For recycled concrete, the situation is slightly different.

When the water binder ratio is higher than 0.4 , the compressive strength of recycled concrete decreases with the increase of water binder ratio, which is basically consistent with that of ordinary concrete; When the water binder ratio is lower than 0.4 , the compressive strength of recycled concrete does not increase effectively with the decrease of water binder ratio. The reason is that when the water binder ratio is high, the strength of cement slurry around recycled aggregate decreases, and the damage of concrete starts from the cracking of cement slurry [9]. When the water binder ratio is low, the strength of cement slurry is relatively high, and the strength of recycled concrete tends to be determined by the strength of recycled aggregate, so that the compressive strength of recycled concrete cannot be improved with the increase of water slurry strength.

Whether recycled fine aggregate or recycled coarse aggregate, whether the aggregate is simple crushing or particle shaping, the relationship between compressive strength and water cement ratio of recycled concrete is similar to that of ordinary concrete. This fully shows that the water cement ratio is a very important factor affecting the compressive strength of recycled concrete. Too large or too small water cement ratio will reduce the compressive strength of recycled concrete. When the water cement ratio is too large, the slurry fluidity of recycled concrete is large, a large amount of water will evaporate when the concrete is hardened, and more pores will be generated in the concrete, which will affect the mechanical properties and 
Article History: Received: 28 October 2021 Revised: 05 December 2021 Accepted: 10 January 2022 Publication: 28 February 2022

durability of recycled concrete. When the water cement ratio is too small, the recycled concrete has poor workability and low fluidity, and the concrete is not easy to be fully hydrated, which has an adverse effect on the compressive strength of recycled concrete [10]. At the same time, when the recycled concrete is hardened, if it meets water again, the unhydrated cement will participate in the hydration reaction again, and the resulting expansion force will crack the structure, reduce the strength, and reduce the impermeability and crack resistance of the recycled concrete structure.

2.Effect of replacement rate of recycled aggregate on strength of recycled concrete

In order to study the variation law of the compressive strength of recycled concrete and the replacement rate of recycled aggregate, the average value of the compressive strength of recycled concrete at 3D, 28d, 56d and 150D with different cementitious materials and the same replacement rate of recycled aggregate (all 35\%) in the test of the research group is drawn, and the influence diagram of the replacement rate of recycled aggregate on the strength of recycled concrete is obtained. The research shows that when the replacement rate of recycled fine aggregate changes from $0 \%, 40 \%, 70 \%$ and $100 \%$, the absolute difference of strength of recycled fine aggregate concrete with different cementitious materials at each age is not more than $10 \mathrm{MPa}$, and the relative difference is less than $15 \%$. It shows that the replacement rate of recycled fine aggregate has no significant effect on the compressive strength of recycled fine aggregate concrete at each age.

When the replacement rate of recycled aggregate exceeds $40 \%$, the compressive strength of recycled coarse aggregate concrete does not increase obviously with the increase of replacement rate of recycled aggregate; However, the $28 \mathrm{~d}$ and $56 \mathrm{~d}$ compressive strength of recycled concrete with $40 \%$ replacement rate of recycled aggregate is significantly higher than that of concrete prepared with natural aggregate. The main reasons why the strength of recycled concrete is higher than that of reference concrete are as follows: first, the properties of high-quality recycled coarse aggregate are improved and the crushing index is significantly reduced after particle shaping; Second, the cement mortar adhered to the surface of recycled coarse aggregate has high water absorption, which reduces the effective water binder ratio when preparing recycled concrete; Third, when the replacement rate of recycled coarse aggregate does not exceed $40 \%$, it has a significant impact on the effective water binder ratio, which can effectively improve the compressive strength of recycled concrete.

\section{BASIC PRINCIPLE OF ARTIFICIAL NEURAL NETWORK}

\section{Overview of artificial neural network technology}

Artificial neural network originated in the late 19th century and has experienced four periods: Enlightenment period, low tide period, revival period and new period. The function of the original artificial neural network is simple, mainly represented by the single-layer 
Article History: Received: 28 October 2021 Revised: 05 December 2021 Accepted: 10 January 2022 Publication: 28 February 2022

perceptron. Because the single-layer perceptron can only carry out linear classification and can't do anything about the linear inseparable input mode, the development of artificial neural network is at a low tide. In 1986, rummerhart et al. Proposed an algorithm to solve the weight correction of multilayer neural network - error back propagation algorithm (BP algorithm for short). It is a breakthrough research achievement of artificial neural network, which adds vitality to artificial neural network and makes it recover and develop comprehensively and rapidly.

Since the 1980s, the rapid development of artificial neural network has been a hot spot in nonlinear scientific research. Its theoretical research results have been widely used in many application fields. Many problems that cannot be solved by traditional information processing methods (such as function approximation, optimization problem calculation, intelligent information processing, etc.) have achieved good results after using neural network. Specifically, neural networks are mainly used in the fields of information processing, automation, economy, medicine and engineering. The engineering fields include automotive engineering, military engineering, chemical engineering and civil engineering. In recent years, Chinese researchers have carried out research on the application of artificial neural network to predict the properties of various types of concrete, such as working and mechanical properties.

2.Structure and main idea of BP network

Back propagation network (BP network for short) is the most widely used and mature neural network model. BP network is a one-way propagation multilayer feedforward neural network, which is generally composed of input layer, hidden layer and output layer. The transfer function from input layer to hidden layer neuron is S-type function, and the input and output quantities are continuous quantities between 0 and 1 . It can realize any nonlinear mapping from input to output. Because the weight adjustment adopts back propagation learning algorithm, it is also often called BP network.

BP network model is a feedforward network model in neural network, which uses error back propagation algorithm as its learning algorithm. It is usually composed of input layer, hidden layer and output layer. The neurons between adjacent layers are fully interconnected and connected with each other through the corresponding network weight vector bin, but there is no connection between the neurons in each layer.

The basic idea of error back propagation algorithm is the least square algorithm. It adopts gradient search technology to minimize the error mean square between the actual output value and the expected output value of the network. The main idea of error back propagation algorithm is to divide the learning process into two stages: the first stage (forward propagation process), which gives the input information, processes it through the input layer through the hidden layer, and calculates the actual output value of neurons; In the second stage (error back propagation process), if the expected output value is not obtained in the output layer, the difference between the actual output and the expected output is recursively calculated layer by 
Article History: Received: 28 October 2021 Revised: 05 December 2021 Accepted: 10 January 2022 Publication: 28 February 2022

layer, and the difference is used to adjust the weight and threshold. The two processes run repeatedly to minimize the error signal until it reaches the allowable error range, then the learning process ends.

\section{ESTABLISHMENT OF STRENGTH PREDICTION MODEL OF RECYCLED CONCRETE}

1.Establishment of strength prediction model of recycled concrete based on BP neural network

Because BP neural network can realize the nonlinear relationship between input data and output data, and has strong prediction ability for variables similar to input data, BP neural network is widely used to solve the problem of nonlinear law.

1) Determination of input and output layer. In the neural network based on BP algorithm, the selection of the number of nodes in the input and output layers often has a great impact on the performance of the network, so the number of nodes in each layer needs to be selected appropriately. The number of neurons in the input and output layers is determined according to the needs of practical problems. The input variable is the influencing factor and the output variable is the assessment index.

2) Determination of the number of hidden layers and nodes. It has been proved theoretically that the network with deviation and at least one S-type hidden layer plus a linear output layer can approximate any rational function. This has actually given us a basic principle for designing BP neural network. Increasing the number of layers can further reduce the error and improve the accuracy, but it also complicates the network, thus increasing the training time of network weights.

3) Selection of network transfer function. Whether it is error approximation or pattern recognition, the neural network must be trained. By consulting a large number of relevant data and experimental examples, it is found that the levengerg Marquardt training method with trainltn training function has high convergence speed, short training time and high accuracy.

4) Initialization of network weights. When designing BP network, when the number of nodes in the input layer, hidden layer and output layer of the network structure and the transfer function between neurons in each layer are determined, the initialization function initff can be used to initialize the BP neural network with up to three layers to obtain the weight and interpretation value of each layer.

5) BP neural network model diagram

The established BP neural network model of recycled concrete is shown in Figure 1 below. 
Article History: Received: 28 October 2021 Revised: 05 December 2021 Accepted: 10 January 2022 Publication: 28 February 2022

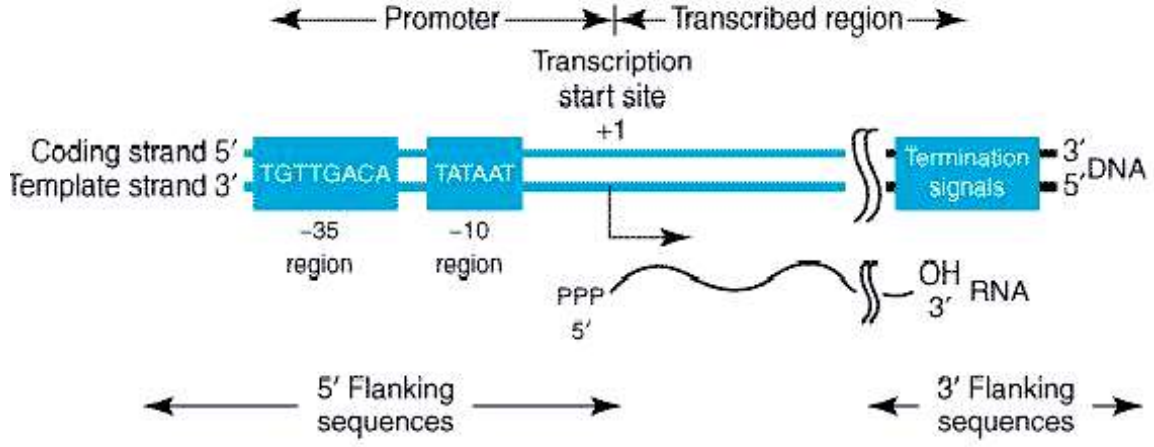

Fig 1: BP network structure of recycled concrete strength prediction model

2.Application of strength prediction model of recycled coarse aggregate concrete based on BP neural network

For each recycled concrete strength prediction model based on BP neural network, 24 additional groups of recycled concrete need to be prepared as test samples. The compressive strength of 24 groups of test samples is predicted by BP neural network model, and the predicted results are compared with the compressive strength of the corresponding age measured in the test, so as to evaluate the performance of the strength prediction model based on BP neural network. Raw materials (including cement, fly ash, coarse aggregate, fine aggregate, recycled coarse aggregate and water reducer) with the same material properties as the collected training samples of recycled coarse aggregate concrete are selected to mix 24 groups of recycled coarse aggregate concrete. After standard curing to a certain age, the compressive strength value is obtained through compressive test as the test sample data. According to the above mix proportion, recycled concrete with slump in the range of 160$200 \mathrm{~mm}$ is prepared respectively. For each mix proportion, three recycled concrete test blocks are formed with triple formwork to test the 3D, 28d and 56d compressive strength of recycled concrete. The compressive strength of the recycled coarse aggregate concrete at the corresponding age is shown in Table 1.

TABLE I. Test value of recycled coarse aggregate concrete test sample

\begin{tabular}{|c|c|c|c|c|c|c|c|}
\hline \multirow{2}{*}{ NUMBER } & \multicolumn{3}{|c|}{ AGE } & \multirow{2}{*}{ NUMBER } & \multicolumn{3}{|c|}{ AGE } \\
\cline { 2 - 5 } \cline { 5 - 8 } & $3 \mathrm{~d}$ & $28 \mathrm{~d}$ & $56 \mathrm{~d}$ & & $3 \mathrm{~d}$ & $28 \mathrm{~d}$ & $56 \mathrm{~d}$ \\
\hline 1 & 25.6 & 43.6 & 52.9 & 13 & 22.1 & 39.6 & 42.9 \\
\hline
\end{tabular}


January-February 2022 Page No.165-172

Article History: Received: 28 October 2021 Revised: 05 December 2021 Accepted: 10 January 2022 Publication: 28 February 2022

\begin{tabular}{|c|c|c|c|c|c|c|c|}
2 & 23.2 & 35.3 & 42.8 & 14 & 24.9 & 45.4 & 52.8 \\
\hline 3 & 23.3 & 41.7 & 45.2 & 15 & 18.4 & 37.4 & 46.4 \\
\hline 4 & 23.2 & 42.5 & 49.9 & 16 & 18.4 & 39.2 & 45.1 \\
\hline 5 & 21 & 38.5 & 45.1 & 17 & 34 & 51.6 & 53.7 \\
\hline 6 & 25 & 41.2 & 46.4 & 18 & 39.5 & 64.1 & 64.9 \\
\hline 7 & 23.8 & 39.1 & 44.1 & 19 & 24.1 & 47.9 & 55.8 \\
\hline 8 & 26.5 & 45.7 & 52.2 & 20 & 25.6 & 43.6 & 58.2 \\
\hline 9 & 26.1 & 49.4 & 54.6 & 21 & 45.7 & 64.1 & 69.1 \\
\hline 10 & 23.7 & 44.7 & 49.4 & 22 & 46.9 & 67 & 74.8 \\
\hline 11 & 23.7 & 43.2 & 50.3 & 23 & 30.7 & 59 & 56.2 \\
\hline 12 & 22.5 & 41 & 47.8 & 24 & 28 & 56.7 & 66.5 \\
\hline
\end{tabular}

The trained BP neural network model was tested with 24 groups of test data, and the values of input variables of recycled coarse aggregate concrete test samples (including 8 parameters: aggregate type, cement, fly ash, water binder ratio, water reducer, coarse aggregate, fine aggregate and aggregate substitution rate) were normalized. Then the normalized test data are brought into the trained strength prediction model of recycled coarse aggregate concrete based on BP neural network, and the simulation results of 24 groups of recycled coarse aggregate concrete test samples at the corresponding age are obtained.

The strength prediction model of recycled coarse aggregate concrete based on BP neural network can successfully establish the nonlinear relationship between recycled concrete mix proportion and its mechanical properties, that is, the strength of corresponding age can be predicted from the given recycled concrete mix proportion. The biggest advantage of BP neural network is that it is good at dealing with a large number of seemingly irregular problems with potential hidden laws between input and output data. By accumulating more data in the longterm application, the network structure can be continuously improved and the network can achieve higher prediction accuracy.

\section{CONCLUSION}

The model of the relationship between each component and strength of recycled concrete based on BP artificial neural network is different from the traditional model in that it has no clear mathematical expression. Therefore, there is no traditional method to simplify the approximate process of the complex relationship between the corresponding components and strength, which reflects its advantages. On the other hand, the establishment of artificial neural network model needs certain training samples. We must improve the quantity and quality of 
Article History: Received: 28 October 2021 Revised: 05 December 2021 Accepted: 10 January 2022 Publication: 28 February 2022

training samples, otherwise the pre side effect of network model will be affected to varying degrees.

\section{REFERENCES}

1. Cai Shaohuai. the Latest Development of Concrete Filled Steel Tubular Structure Technology in China. Acta Civil Engineering, 1999, 32 (004): 16-26

2. Guo Zhenhai, Wang Chuanzhi. Study on Strength and Failure Criterion of Concrete Under Multiaxial Stress. Acta Civil Engineering, 1991, 024 (003): 1-14

3. Nie Jianguo, Yu Zhiwu. Research and Application of Steel-concrete Composite Beams in China. Acta Civil Engineering, 1999, 32 (2): 3-8

4. Zhou Junsheng, Lou Zhuanghong. Present Situation and Development Trend of Long Span Prestressed Concrete Continuous Rigid Frame Bridge. Acta Highway Sinica, 2000, 13 (1): 9-13

5. Lu Xilin, Lu Weidong. Experimental Study on Seismic Behavior of Concrete Filled Steel Tubular Columns Under Cyclic Loading. Acta Architectural Structures, 2000 (2):12-19

6. Ma Huaifa, Chen Houqun, Li Baokun. Numerical Simulation of Mesostructure of Concrete Specimen. Acta Hydrologica Sinica, 2004, 35 (10): 27-35

7. Zhu Bofang. Implicit Method for Creep Stress Analysis of Concrete Structures. Acta Hydrologica Sinica, 1983, 5: 42-48

8. Jiang Chuanliang, Xian Qiaoling. Nonlinear Finite Element Analysis of Reinforced Concrete Structures. Science and Technology and Engineering, 2005, 5 (017): 1323-1324

9. Niu Bin. Analysis of Flexural Behavior of Externally Prestressed Concrete Beams. Acta Civil Engineering, 1999 (04): 37-44

10. Wu Gang, an Lin, Lv Zhitao. Experimental Study on Shear Strengthening of Reinforced Concrete Beams with Cfrp Sheets. Building Structures, 2000 (07): 3-6 + 10 